\title{
Intervenção Coronária Percutânea em Idosos
}

\author{
Ver artigos relacionados \\ nas páginas 17 e 24
}

\author{
Otavio C. E. Gebara ${ }^{1,2}$, Mauricio Wajngarten ${ }^{1}$
}

O envelhecimento populacional se apresenta como um grande desafio para a comunidade médica. A despeito dos constantes avanços no diagnóstico, da compreensão dos mecanismos e do tratamento, a doença arterial coronária ainda é a causa mais importante de morbidade e mortalidade em indivíduos com mais de 65 anos de idade. Nas últimas décadas, desde sua descrição, a intervenção coronária percutânea tem se mostrado uma opção terapêutica cada vez mais utilizada em idosos.

Em comparação à população mais jovem, os pacientes idosos submetidos a procedimento de revascularização percutânea apresentam mais frequentemente doença difusa, disfunção ventricular, síndromes coronárias agudas e comorbidades como insuficiência renal, acidente vascular encefálico e doença pulmonar obstrutiva crônica, colocando-os em situação clínica mais desfavorável. Essas características tornam a avaliação de resultados de intervenções nessa faixa etária mais dificultosa. Soma-se a isso o fato de que os estudos clínicos controlados, que produzem as melhores evidências científicas, geralmente não incluem indivíduos com as características clínicas mais graves (extremos de idade e comorbidades), o que torna as diretrizes pouco aplicáveis na prática clínica, em que a individualização passa a ser indispensável.

Nesta edição da Revista Brasileira de Cardiologia Invasiva temos dois estudos interessantes de intervenção coronária percutânea em pacientes com mais de 80 anos. Thomas Júnior et al. ${ }^{1}$ avaliaram retrospectivamente os resultados hospitalares da intervenção coronária percutânea primária em 34 pacientes com mais de 80 anos com infarto do miocárdio, e os compararam com um grupo de 394 pacientes com menos de 80 anos de idade. Observou-se que não houve diferença quanto à taxa de sucesso angiográfico $(97,1 \%$ vs. $96,5 \%$; $P>0,99$ ), porém o grupo com mais de 80 anos apresentou mortalidade hospitalar mais elevada $(11,8 \%$ vs. $2,3 \% ; P=0,014)$. O grupo mais idoso tinha proporção maior de mulheres e pior função ventricular. Outras complicações foram semelhantes àquelas observadas em pacientes com menos de 80 anos. Já Pimentel Filho et al. ${ }^{2}$ avaliaram o sucesso angiográfico e a evolução clínica a longo prazo (36 meses) de um grupo de 31 pacientes nonagenários submetidos a intervenção coronária percutânea sem infarto agudo do miocárdio. Os autores demonstraram pior sucesso angiográfico nos pacientes nonagenários $(87 \%$ vs. $95,1 \%$ ), assim como maior incidência hospitalar de complicações vasculares maiores, óbito e infarto do miocárdio, e pior evolução clínica tardia (sobrevivência livre de eventos de $68 \%$ vs. $91 \%$ no grupo mais jovem). A pior condição cardiológica e a presença de comorbidades foram os preditores de eventos no seguimento. Nos nonagenários houve maior prevalência de doença multiarterial e revascularização prévia e pior taxa de revascularização completa. Nesse grupo de pacientes existia alta prevalência de doença pulmonar obstrutiva crônica (um terço da amostra), insuficiência renal e neoplasias.

Estudando pacientes de faixa etária semelhante, Lemos et al. ${ }^{3}$ avaliaram, também em nosso meio, a evolução tanto hospitalar como tardia (dois anos) de 246 pacientes com mais de 80 anos de idade submetidos a intervenção coronária percutânea. Esses autores concluíram que essa população apresentava mortalidade relativamente alta (sobrevida de $76 \%$ em dois anos), mas os maiores preditores de mau prognóstico eram a presença de infarto do miocárdio na admissão e a presença de doença triarterial (quando ambos estavam presentes a sobrevida caiu para $56 \%$ em dois anos).

O Trial of Invasive Versus Medical Therapy in Elderly Patients (TIME) ${ }^{4}$ foi o primeiro estudo clínico prospectivo e controlado que comparou a estratégia invasiva (intervenção coronária percutânea ou cirurgia de revascularização) com tratamento medicamentoso otimizado em pacientes com idade igual ou superior a 75 anos. Os autores concluíram que os sintomas, a

\footnotetext{
1 Divisão Clínica de Cardiogeriatria - Instituto do Coração do Hospital das Clínicas da Faculdade de Medicina da Universidade de São Paulo (InCor/HCFMUSP) - São Paulo, SP, Brasil.

${ }^{2}$ Hospital Total Cor de São Paulo - São Paulo, SP, Brasil.

Correspondência: Otavio C. E. Gebara. InCor - Av. Dr. Enéas Carvalho de Aguiar, 44 - Cerqueira César - São Paulo, SP, Brasil - CEP 05403-900.

E-mail: carotavio@uol.com.br

Recebido em: 2/3/2010 • Aceito em: 4/3/2010
} 
qualidade de vida, a mortalidade e a incidência de infarto do miocárdio foram semelhantes no primeiro ano. Porém, 43\% dos pacientes selecionados para tratamento clínico foram submetidos a procedimentos de revascularização por instabilidade clínica no seguimento, demonstrando que a indicação de terapêuticas não é tarefa simples nessa faixa etária.

O grande valor dos estudos de intervenção coronária percutânea em extremos de idade publicados nesta edição da Revista Brasileira de Cardiologia Invasiva está na demonstração da segurança do procedimento e no prognóstico clínico a longo prazo.

É interessante a utilização, no estudo de Pimentel Filho et al., ${ }^{2}$ do índice de Katz, que mensura o grau de independência funcional para atividades da vida diária, demonstrando que esse grupo era formado por nonagenários com bom grau de independência. A inclusão dessa variável, na análise de Pimentel et al., ${ }^{2}$ aponta para questões de suma importância quando se avalia qualquer terapêutica em idosos, principalmente nos muito idosos, como é o caso desses dois estudos.

Na tentativa de tornar tais avaliações mais objetivas, escores têm sido propostos. Entre eles, destacamse, para complexidade angiográfica, o escore SYNTAX ${ }^{5,6}$ e, para avaliação de comorbidades, o escore de Charlson. ${ }^{7}$ Embora índices e escores tenham demonstrado eficiência em estudos importantes, não devem dispensar a análise individualizada e criteriosa realizada por intervencionistas, cirurgiões e clínicos, com a colaboração eventual de outros profissionais da saúde. Finalmente, salientamos que a conduta deve se basear, além do exposto, na expectativa e nas preferências de pacientes e familiares. Evidentemente, isso exige diálogo, paciência e muita sensibilidade.

\section{CONFLITO DE INTERESSES}

Os autores declararam inexistência de conflito de interesses relacionado a este manuscrito.

\section{REFERÊNCIAS}

1. Thomas Júnior NR, Cantarelli MJC, Castello Junior HJ, Gioppato S, Gonçalves R, Guimarães JBF, et al. Resultados hospitalares da intervenção coronária percutânea primária no infarto agudo do miocárdio em pacientes com mais de 80 anos. Rev Bras Cardiol Invasiva. 2010;18(1):17-23.

2. Pimentel Filho WA, Carvalho $C A$, Pelegrini $M$, Wellington BC, Bocci EA, Soares Neto MC, et al. Intervenção percutânea coronária em pacientes nonagenários. Rev Bras Cardiol Invasiva. 2010;18(1):24-9.

3. Lemos PA, Campos CAH, Ribeiro EE, Falcão JLAA, Perin MA, Kajita LJ, et al. Incidência e preditores de óbito precoce e tardio em octagenários brasileiros tratados com intervenção coronária percutânea. Rev Bras Cardiol Invasiva. 2007;15(1):31-4.

4. Pfisterer $M$, Buser P, Osswald S, Allemannn U, Amann W, Angehrn W, et al.; for the Trial of Invasive versus Medical Therapy in Elderly Patients (TIME) Investigators. Outcome of elderly patients with chronic symptomatic coronary artery disease with an invasive versus optimized medical treatment strategy: one year results of the randomized TIME trial. JAMA. 2003;289(9):1117-23.

5. Boden WE, O'Rourke RA, Teo KK, Hatigan PM, Maron DJ, Kostuk WJ, et al. Optimal medical therapy with or without PCl for stable coronary artery disease. N Engl J Med. 2007; 356(15):1503-16.

6. Serruys PW, Morice MC, Kappetein AP, Colombo A, Holmes DR, Mack MJ, et al. Percutaneous coronary intervention versus coronary-artery bypass grafting for severe coronary artery disease. N Engl J Med. 2009;360(10):961-72.

7. Charlson M, Szatrowski T, Peterson J, Gold J. Validation of a combined comorbidity index. J Clin Epidemiol. 1994;47(11): 1245-51. 